\title{
Action of centrally mediated autonomic stimulation on human upper gastrointestinal transit: a comparative study of two stimuli
}

\author{
J D O'BRIEN, D G THOMPSON, W R BURNHAM, J HOLLY, \\ AND E WALKER
}

From the Departments of Gastroenterology and Chemical Pathology, The London and Oldchurch Hospital, London

SUMmARY Two well established experimental stressors, hand immersion in cold water, and mental stimulation with dichotomous listening, were applied to 37 normal subjects after the ingestion of a standard meal. Orocaecal transit was measured by serial exhaled breath hydrogen sampling. Cold water significantly delayed transit compared with warm water control (warm water $71.8 \pm 3.6 \mathrm{mins} v$ cold water $93.2 \pm 5.7$ mins $\mathrm{p}<0.01$ ), with significant rises in blood pressure pulse rate perceived discomfort and plasma catecholamines. In contrast mental stimulation was unaccompanied by any change in transit (control; 67.4 $\pm 4.7 v$ test $64.3 \pm 5.3$ mins $p>0.1$ ) despite a significant rise in pulse rate, skin conductance and plasma catecholamines. Repeated cold water immersion studies in eight individuals produced consistent orocaecal transit and autonomic responses, whereas mental stimulation showed reduced autonomic responses on repeat testing, suggesting that tolerance to the stimulus had occurred. The results of these studies show stimulus specific gastrointestinal response patterns to autonomic stimuli, and appear to have important implications for the design of future studies of human gastrointestinal autonomic physiology and for the investigation of patients with stress related gut dysfunction.

The effects of centrally mediated disturbance of human gastrointestinal autonomic nervous function remain poorly understood despite the considerable clinical suspicion of a relationship between stress and the development of gastrointestinal disease. For more than a century studies have suggested that stress might disturb gut function ${ }^{1.3}$ but data from controlled clinical experiments to support this contention remain far from conclusive. In recent years improvements in the techniques available for the study of human gut function has enabled a return to the subject to be made, and objective studies of the functional interrelationships between the central nervous system and the gut have been reexamined. ${ }^{+11}$ While these recent studies have undoubtedly advanced our knowledge, the diversity

Address for correspondence: Dr D G Thompson, Senior Lecturer in Medicine, Department of Medicine. Hope Hospital, Manchester M6 8HD.

Received for publication 5 December 1986. of experimental design has made understanding difficult. Researchers using different experimental protocols and using different techniques for autonomic stimulation have shown conflicting responses. Stress induced experimentally by immersion of a hand into cold water has been shown to disturb gastric emptying and acid secretion ${ }^{+}$and to inhibit antral motor activity. ' Labyrinthine stimulation has been shown to produce similar effects on gastric function ${ }^{6}$ but a different pattern of small intestinal response occurs. In contrast, mental stimulation tasks such as dichotic listening, ' delayed auditory feedback ${ }^{*}$ or high decibel noise" are reported to disturb fasting small intestinal motility with an inhibition of phase III activity ${ }^{7}$ and also accelerate orocaecal transit, but seem to be without effect on gastric emptying."

In many of these recent studies, an implicit assumption has been, that stress is a single definable entity and that the autonomic responses to different 
disturbing stimuli would be similar. Although initially considered in this way," more recent data indicate that autonomic responses are much more stimulus specific, ${ }^{12}$ so that the results of experiments done with one stimulus might be quite different from those done with another. Because direct comparison of gastrointestinal responses to different experimental stimuli is lacking, we undertook a detailed study of upper gastrointestinal transit responses to two commonly used experimental stressors, cold pain and mental stimulation. The results of these studies show stimulus specific patterns of response which have important implications for the design of future studies of human gastrointestinal autonomic physiology and for the investigation of patients with possible stress related gut dysfunction.

\section{Methods}

\section{SUBJECTS}

All the experiments described below were performed on 37 healthy adult volunteers (aged 19-51 years) who gave their informed written consent before participation. All experimental protocols related to the studies were presented to, and approved by, The London Hospital Ethics Committee.

\section{OROCAECAL TRANSIT}

To provide an index of upper gastrointestinal function, the orocaecal transit of the poorly absorbed carbohydrate lactulose was chosen. ${ }^{13}$ This technique (which relies upon the detection of a rise in hydrogen concentration in the exhaled breath at the time of carbohydrate arrival at the caecum) was selected because of its simplicity and freedom from physical discomfort. In addition, its lack of radiation exposure or other hazard made it particularly suitable for repeated studies in normal volunteers which were necessary for assessment of inter- and intraindividual responses, to a stimulus and for the comparison of responses to different stimuli within individuals.

For all the experiments described, a standard method for orocaecal transit measurement was used, the experimental conditions being chosen after the conduct of the series of pilot studies described below.

\section{Test meal}

The test meal was developed from one previously used for similar studies ${ }^{\text {th }}$ and consisted of a $400 \mathrm{ml}$ liquid mixed nutrient meal (Chicken soup $255 \mathrm{Kcal}$, Heinz Ltd.) Because ingestion of this meal was not followed by a breath hydrogen rise, the poorly absorbed carbohydrate, lactulose (Duphalac, Duphar Ltd.) was added. To determine the optimal quantity of lactulose nine volunteers ingested a series of soup meals, containing either 10,20 or $30 \mathrm{~g}$ lactulose. As the quantity of lactulose in the meal increased, the transit time decreased from $93 \cdot 3 \pm 9 \cdot 8$ mins (mean \pm SEM) with $10 \mathrm{~g}$ to $50 \cdot 6 \pm 5 \cdot 2$ min with 30 g. This transit reduction was, however, accompanied by a tendency to develop abdominal discomfort and diarrhoea at the highest dose. A $20 \mathrm{~g}$ dose of lactulose was therefore selected for the studies, being associated with a transit time of convenient length without the production of abdominal symptoms. Using this dose of lactulose on three occasions, eight individuals showed a mean overall transit time of $64.0 \pm 3.8 \mathrm{~min}$ with a mean individual coefficient of variation of $9 \cdot 1 \%$.

\section{Measurement of exhaled breath hydrogen concentration}

All volunteers were trained in the technique of exhaled breath sample collection ${ }^{14}$ before undertaking the studies. During each study subjects collected samples of exhaled breath at five minute intervals (and at two minute intervals when hydrogen values appeared to become raised). The hydrogen concentration in each sample was measured using a commercially available hydrogen sensitive electrochemical cell (GMI Ltd. Renfrew, Scotland) which provided a digitial reading of hydrogen concentration in parts per million ( $\mathrm{ppm}$ ) with an overall accuracy of $\pm 98 \% .{ }^{15}$

\section{TEST STIMULI}

\section{Hand Immersion in cold water}

The procedure used was identical to that developed and used in previously reported studies. ${ }^{+16}$ Subjects placed their non-dominant hand into an ice/water mixture for one minute and then removed it for 15 seconds. This cycle was repeated until 20 minutes had elapsed.

In a previously reported study $y^{4}$ this procedure was found to be universally uncomfortable and to produce measurable autonomic changes both during the procedure and for up to 15 minutes after cessation of the immersion, the result of discomfort upon hand rewarming. This poststimulation response was confirmed in a series of pilot studies on 12 normal volunteers; the mean time to cessation of discomfort after completion of immersion being 13 minutes, giving an overall duration of discomfort of $30-35$ minutes.

For the control studies an identical hand immersion procedure was used but the temperature of the water was maintained at $37^{\circ} \mathrm{C}$.

\section{Mental stimulation}

A method based on the previously developed 
dichotic listening test ${ }^{717}$ was used to induce mental arousal. During the test, subjects were exposed to dissimilar narrative monologues delivered, one to each ear, through headphones from a dual channel tape recorder. The recipient was required to repeat aloud one of the monologues whilst ignoring the other, alternating between monologues every minute. Because previous studies ${ }^{7}$ had shown wide inter-individual variability in the magnitude and duration of the autonomic responses to this stimulus, the technique was modified by the addition of mental arithmetic tasks to increase its intensity. Using this modified method, it proved possible to disturb the autonomic nervous system for at least thirty minutes. In contrast to hand immersion in cold water, however, the autonomic responses quickly returned to prestimulation values, within five minutes of stimulus cessation.

For the control stimulus, subjects listened to light music through stereo headphones.

\section{Duration of application of the two stimuli}

Because of the differences in duration of the poststimulus response, the mental task was applied for 30 minutes while the hand immersion was applied for 20 minutes. This ensured that with both stimuli an autonomic response with a duration of 20-35 minutes was obtained.

\section{Extra-intestinal autonomic responses to the stimuli}

To provide an assessment of autonomic nervous response, independent of gastrointestinal function, serial measurements of brachial arterial blood pressure (using a random-zero sphygmomanometer) and radial artery pulse rate (by palpation) were made, together with serial measurements of digitial skin conductance, an index of sympathetically induced sweat production (Skin conductance meter, Electronic Development, Hampton, Middlesex).

\section{Perception of stimulus severity}

To obtain an index of the magnitude of the discomfort experienced during each experiment all subjects were requested to provide repeated estimates of their level of discomfort, at five minute intervals, using a simple linear scale ranging from 0 (complete comfort) to 10 (intolerable discomfort).

\section{PLASMA CATECHOLAMINE MEASUREMENT}

Venous blood samples were withdrawn via an indwelling antecubital vein cannula, inserted 30 minutes before the start of the study, from the first seven subjects in each of the two studies, at 10 minute intervals commencing 10 minutes before application of the stimulus and containing for $\mathbf{4 0}$ minutes after its cessation. After collection, all samples were centrifuged and stored in liquid nitrogen until analysis. Plasma catecholamines after isolation on alumina were separated by ion pair reverse phase high pressure liquid chromatography and quantified with an electrochemical detector (Model 5100A Coulochem, Environmental Sciences Associates Inc, Bedford, Mass, USA) operated in the redox mode. The minimum detectable limits of the assay were approximately 5 pg noradrenaline and adrenaline per $\mathrm{ml}$ of plasma. At a plasma noradrenaline concentration of $300 \mathrm{pg} / \mathrm{ml}$ the intra-assay and interassay coefficients of variation were $3 \cdot 2 \%$ and $6.0 \%$ respectively, and at a plasma adrenaline concentration of $60 \mathrm{pg} / \mathrm{ml}$ the corresponding coefficients of variation were $6 \cdot 7 \%$ and $9 \cdot 1 \%$.

EXPERIMENTAL PROCEDURE

The experimental protocol outlined in Figure 1 was used for all the studies. Subjects arrived at the clinical study laboratory at 9 am after an overnight fast of at least 15 hours. During each study they reclined on a hospital bed with their upper body supported by pillows at an angle of 30 degrees to horizontal and avoided all activities known to adversely influence the accuracy of breath hydrogen measurement ${ }^{14}$.

Twenty three subjects carried out the test and control hand immersion studies in a randomised order on separate days, while 22 subjects did the test and control mental stimulation studies. Eight subjects undertook both experiments. None of the subjects had previously experienced the stimulus before conduct of the study.

\section{REPRODUCIBILITY STUDIES}

To evaluate the reproducibility of the response to the two stimuli, the test and control experiments were repeated three times in 13 subjects (eight with hand immersion and five with mental stimulation). The order in which the test and control studies were done was randomised for each individual.

INDIVIDUAL RESPONSES TO THE TWO STIMULI To evaluate individual differences in the responses to the two stimuli, eight individuals did both the hand immersion and mental stimulation experiments in a randomised order.

STATISTICAL ANALYSIS

For the purpose of data display, group means and standard errors of the mean (SEM) have been used. In order to avoid the assumption of normality of data distribution, the non-parametric Wilcoxon's matched pairs signed-rank-test ${ }^{18}$ was used for assessment of probability, in addition to a paired $t$ test, a $p$ value of $<0.05$ for both tests being taken to indicate that the observed differences were unlikely 
$\left.\begin{array}{r}\text { Warm water } 37^{\circ} \mathrm{C} \\ \text { Restful music }\end{array}\right\}$

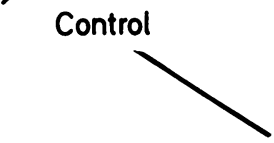

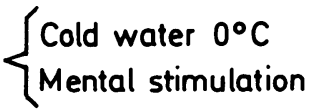

to have been chance related. Correlation coefficients were calculated with the Kendall Rank Correlation Test. ${ }^{18}$

\section{Extra-intestinal responses}

For the analysis of these data the mean of the values for each variable were calculated over the 30 minute prestimulation period and during the 30 minute stimulation period. The difference between these two values was then compared for the test and the control studies and this difference was used as an index of the magnitude of the response to the stimulus for that individual.

\section{Orocaecal transit}

The arrival of meal marker at the caecum was taken to have occurred at the onset of a rise in exhaled breath hydrogen. For the purpose of data analysis, a hydrogen rise was defined as having occurred when the hydrogen concentration exceeded twice the mean pre-meal ingestion value. This definition was chosen, after preliminary inspection of a number of data sets, because it offered a simple, conservative, end point which reduced the possibility of erroneous interpretation due to baseline variation. Use of other end points - for example, estimated time of onset of a rise, time for the rise to exceed $20 \mathrm{ppm}$, produced greater end point variability than the chosen method, but did not influence the significance of the results. The effect of a test stimulus on transit was assessed by determining the difference between test and control values for each individual and then determining the significance of these differences for the group.

\section{Results}

HAND IMMERSION STUDIES

Twenty two of the 23 volunteers reported that cold water immersion was an unpleasant stimulus, but all were able to complete the required 20 minute stimulation period. As in the pilot studies, discomfort persisted after cessation of the immersion for $13 \pm 2$ minutes (mean $\pm \mathrm{SEM}$ ) as hand rewarming occurred. During the warm water immersion experiments no discomfort was experienced by any subject.

\section{Extra-intestinal responses}

These data are shown in Figure 2.

\section{Pressor response}

Cold water immersion produced a highly significant $(p<0.001)$ rise in systolic pressure (mean rise $20.4 \pm 2.1 \mathrm{mmHg}$ ) and diastolic pressure (mean rise $17.8 \pm 1.4 \mathrm{mmHg}$ ) during the stimulation period.

\section{Pulse rate}

The pulse rate was significantly raised by the test stimulus with a mean pulse rise of $4 \cdot 8 \pm 1 \cdot 1$ beats $/ \mathrm{min}$ $(p<0 \cdot 01)$. 


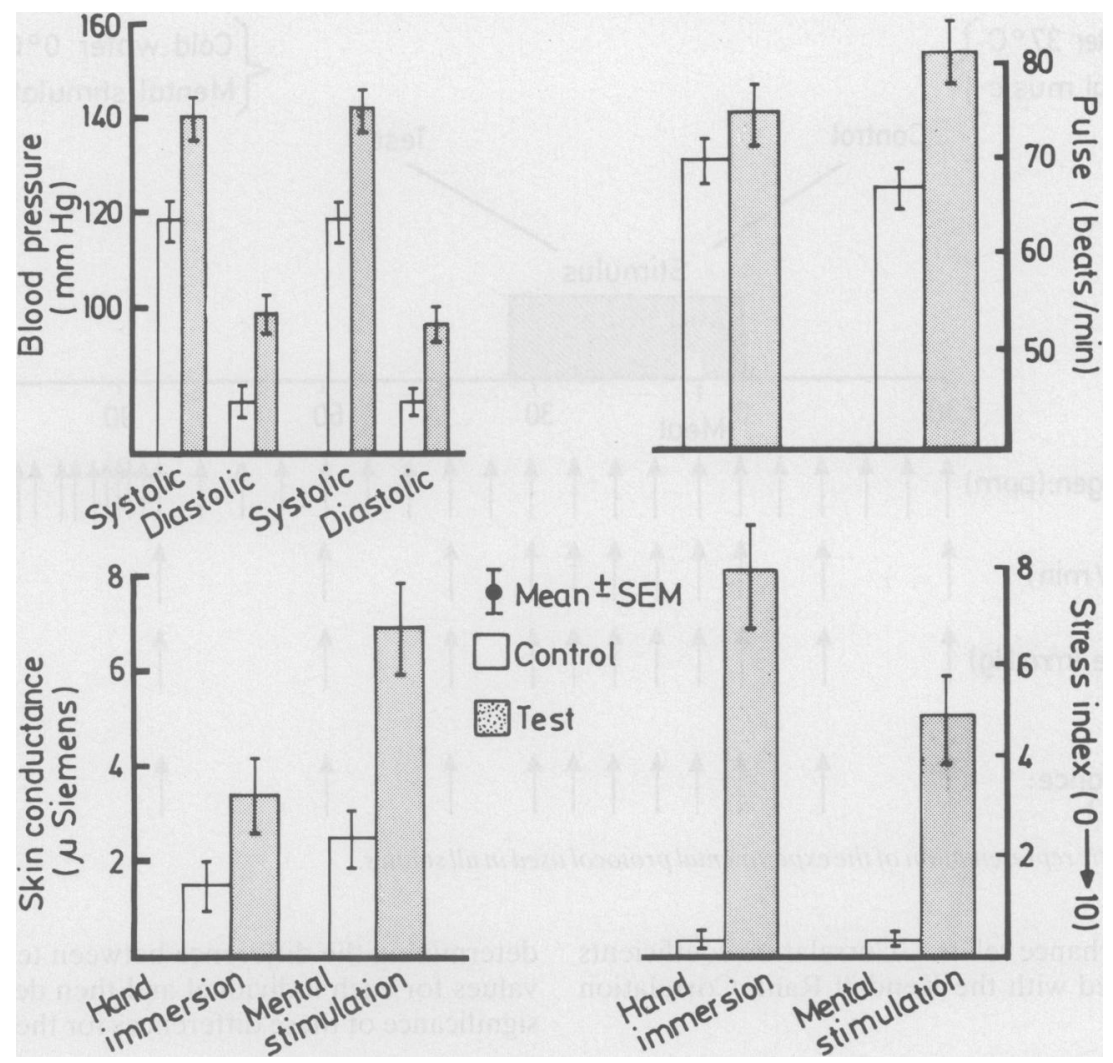

Fig. 2 Extraintestinal responses to hand immersion in cold water and to mental stimulation. Open columns represent the mean $( \pm S E M)$ values for the control stimulus. Hatched columns represent the values for the test stimulus.

\section{Skin conductance}

There was a small but not significant $(\mathrm{p}>0 \cdot 1)$ rise in skin conductance (mean rise $1.7 \pm 0.9 \mu$ Siemens).

\section{Plasma catecholamines (Fig. 3)}

A consistent rise in plasma noradrenaline over control values $(166 \cdot 2 \pm 29.7 \mathrm{pg} / \mathrm{ml} \mathrm{p}<0.05)$, and a consistent rise in plasma adrenaline of $(45 \cdot 2 \pm 14 \cdot 1$ $\mathrm{pg} / \mathrm{ml} \mathrm{p}<0.05$ ) accompanied the test stimulus.

\section{Orocaecal transit (Fig. 4)}

The time to onset of the breath hydrogen rise was delayed by cold water immersion in 18 of the 23 subjects studied. Overall, the mean delay was 21.4 minutes (93.2 \pm 5.7 minutes cold water $v 71.8 \pm 3.6$ min warm water $\mathrm{p}<0.01)$. The magnitude of this orocaecal transit delay correlated with the observed rises in systolic blood pressure $(r=0.33 p<0.05)$ and diastolic blood pressure $(r=0.33 p<0.05)$, but not for other autonomic variables or catecholamine responses.

MENTAL STIMULATION STUDIES

All 22 subjects completed the 30 minute time period and all reported the stimulus to be disturbing. The control stimulus in contrast, was unaccompanied by any mental pertubation.

\section{Pressor response}

A significant rise in both systolic and diastolic blood pressure accompanied test stimulation $(p<0.001)$ (Fig. 2). The magnitude of this response was similar to the cold water immersion experiment, with a mean systolic pressure rise of $23.8 \pm 2.2 \mathrm{mmHg}$ and mean diastolic pressure rise of $15.0 \pm 1.4 \mathrm{mmHg}$. After cessation of the stimulus blood pressure rapidly returned to prestimulation levels (with a mean time to recovery of $6 \cdot 1 \pm 1$ mins).

\section{Pulse rate (Fig. 2)}

All subjects developed a tachycardia during the test stimulus, the mean increase in pulse rate being $14 \cdot 1 \pm 1 \cdot 7$ beats/minute. $(p<0 \cdot 001)$.

Skin conductance (Fig. 2)

Skin conductance rose during the test stimulus, the 

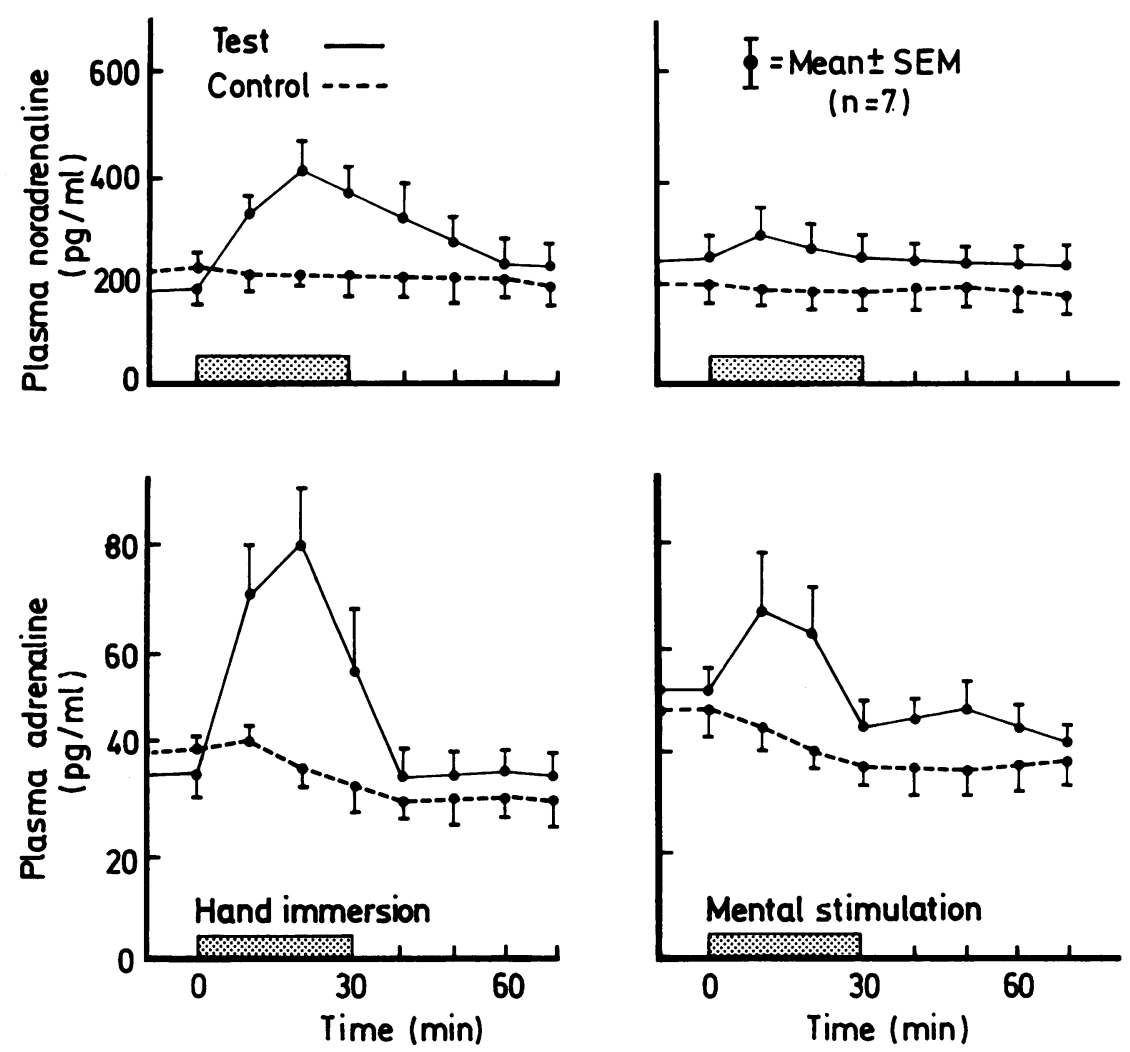

Fig. 3 Plasma catecholamine responses to the two sets of test and control stimuli are shown.

magnitude of this change compared with the control stimulus being, $4 \cdot 6 \pm 1 \cdot 3 \mu$ Siemens $(p<0 \cdot 05)$.

\section{Plasma catecholamines (Fig. 3)}

Mental stimulation was associated with a small rise in plasma noradrenaline over control stimulation $(1 \pm 8.6 \mathrm{pg} / \mathrm{ml} \mathrm{p}>0 \cdot 1)$ while the mean change in plasma adrenaline was $5 \cdot 3 \pm 4 \cdot 9 \mathrm{pg} / \mathrm{ml}(\mathrm{p}>0 \cdot 1)$.

\section{Orocaecal transit (Fig. 4)}

Mental stimulation exerted no significant effect upon orocaecal transit of the test meal compared with control stimulation, the mean time to a breath hydrogen rise for the test experiments being $64 \cdot 3 \pm 5 \cdot 3$ minutes compared with $67 \cdot 4 \pm 4 \cdot 7$ minutes for the control experiments $(p>0 \cdot 1)$.

\section{REPEAT STUDIES}

The results of these studies are shown in Figure 5. The three cold water stimulation studies induced similar autonomic responses on all occasions and were without percepitible difference in discomfort between the first and the third study. The delaying effect on transit was similarly maintained.
In contrast, the repeated mental stimulation studies showed a reduction in the perceived discomfort, with a progressive decline in the magnitude and duration of the autonomic responses with the order of experiment, suggesting that tolerance to the stimulus had occurred.

INDIVIDUAL DIFFERENCES IN RESPONSE TO THE TWO STIMULI

The results of these studies are shown in Figure 6. In the eight subjects who did both experiments, the pressor responses to the two stimuli were very similar with a mean systolic blood pressure rise of $19 \cdot 8 \pm 2 \cdot 6$ $\mathrm{mmHg}$ for hand immersion and $19 \cdot 4 \pm 2 \cdot 1 \mathrm{mmHg}$ for mental stimulation. No consistent differences being found for the group $(p>0 \cdot 1)$.

In contrast, consistent differences were found between the other autonomic variables. Mental stimulation induced a greater rise in pulse rate, with a mean rise of $16 \cdot 5 \pm 3.5$ beats per minute, compared with hand immersion, $5 \cdot 6 \pm 1 \cdot 2$ beats per minute $(p<0.05)$. Skin conductance responses were also consistently different between the two stimuli with a response of $2 \cdot 1 \pm 2 \cdot 8 \mu \mathrm{Siemens}$ for hand immersion 


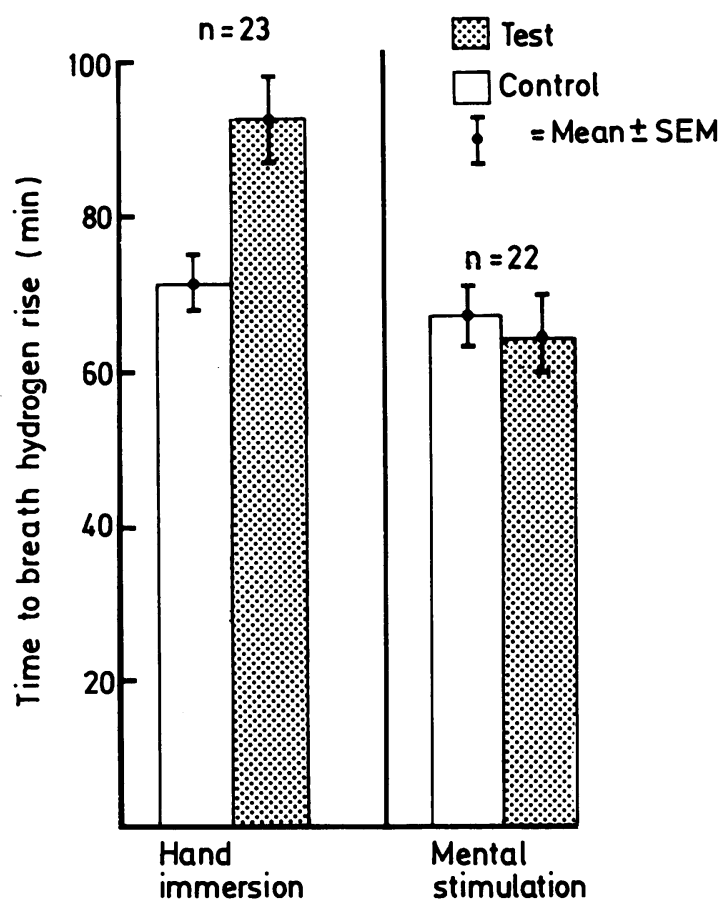

Fig. 4(a) Orocaecal transit to the two experimental stimuli. Hand immersion in cold water significantly delayed transit but mental stimulation exerted no effect.

compared with a response to mental stimulation of $6 \cdot 1 \pm 2 \cdot 1 \mu$ Siemens. $(p<0 \cdot 05)$. Perceived discomfort was greater for hand immersion $8 \cdot 3 \pm 0 \cdot 3$ than for mental stimulation $5 \cdot 6 \pm 0 \cdot 4(\mathrm{p}<0 \cdot 05)$.

As in the unpaired experiments, the transit responses to the two stimuli also differed. Hand immersion produced a consistent delay in orocaecal transit (mean delay $20 \cdot 1 \pm 9.5$ mins $\mathrm{p}<0.05$ ), whereas mental stimulation had no significant effect on transit (mean delay $4 \cdot 6 \pm 12 \cdot 1 \mathrm{~min} p>0 \cdot 1$ ).

\section{Discussion}

The classical concept of autonomic nervous system function proposed by Cannon ${ }^{19}$ was based upon the existence of two mutually opposing systems, the sympathetic and the parasympathetic. In health, it was considered that a harmonious balance existed between these two forces, while a disturbance of autonomic function during stress, resulted in sympathetic stimulation producing physiological and metabolic changes which prepared the body for 'fight or flight'. Until recently this hypothesis served physiological and pharmacological research well and explained most of the known actions of the auto- nomic nervous system. In recent years, however, with the discovery of autonomic pathways which are neither adrenergic nor cholinergic ${ }^{211}$ the naievity of this concept has been recognised. Even the classical sympathetic and parasympathetic nerves are probably associated with the release of peptidergic and purinergic neurotransmitters, ${ }^{21}$ which can themselves modulate the normal autonomic response. Other previously unknown stress related influences on autonomic function include endogenous opiate release from the pituitary ${ }^{22}$ and adrenal, ${ }^{23}$ together with changes in the levels of many other classical hormones, ${ }^{24}$ though which of these responses are primary effects of stress and which are secondary to sympathetic stimulation is difficult to determine. Thus the initially 'simple' autonomic nervous system is now recognised to be highly complex and potentially capable of exerting a wide range of response patterns to stress. While psychophysiologists have recognised such variation ${ }^{12.5}$ the responses of the gut have not previously been considered to be so affected. Our results which show different gastrointestinal as well as extra-intestinal responses to two carefully standardised stimuli now indicate a similar degree of stimulus specificity.

The principal autonomic response to hand immersion in cold water is generally considered to be sympathetic stimulation. ${ }^{26}{ }^{27}$ The rapidity of onset and pattern of the autonomic response, together with the marked rise in circulating noradrenaline indicate that the major pathway for this sympathetic effect is neural rather than hormonal via adrenaline release. It seems likely therefore, that the gastrointestinal response to the stimulus which results in a marked transit delay is also a function of noradrenergic sympathetic stimulation. Previous investigations into the effect of cold stimulation on the upper gut have shown inhibition both of gastric emptying ${ }^{4}$ and of antral motility, ${ }^{5}$ both of which might contribute to the transit disturbance. Other possible contributory effects of sympathetic stimulation could include inhibition of pancreatic ${ }^{+}$and small intestinal ${ }^{2 \times 29}$ secretion.

Because noradrenaline stimulates alpha and beta 1 adrenoreceptors ${ }^{31}$ the gastrointestinal response could be mediated via either or both receptors. Present pharmacological evidence indicates that both receptor subtypes are present in mamalian gut, with alpha adrenoreceptors located principally in the myenteric plexus and beta adrenoreceptors on intestinal smooth muscle. ${ }^{3132}$ It is impossible from our data to make further statements about the mechanism with any certainty, but evidence from other studies appears to indicate that a beta adrenergic pathway may be of prime importance, as gastric emptying of a liquid meal such as that used in our 

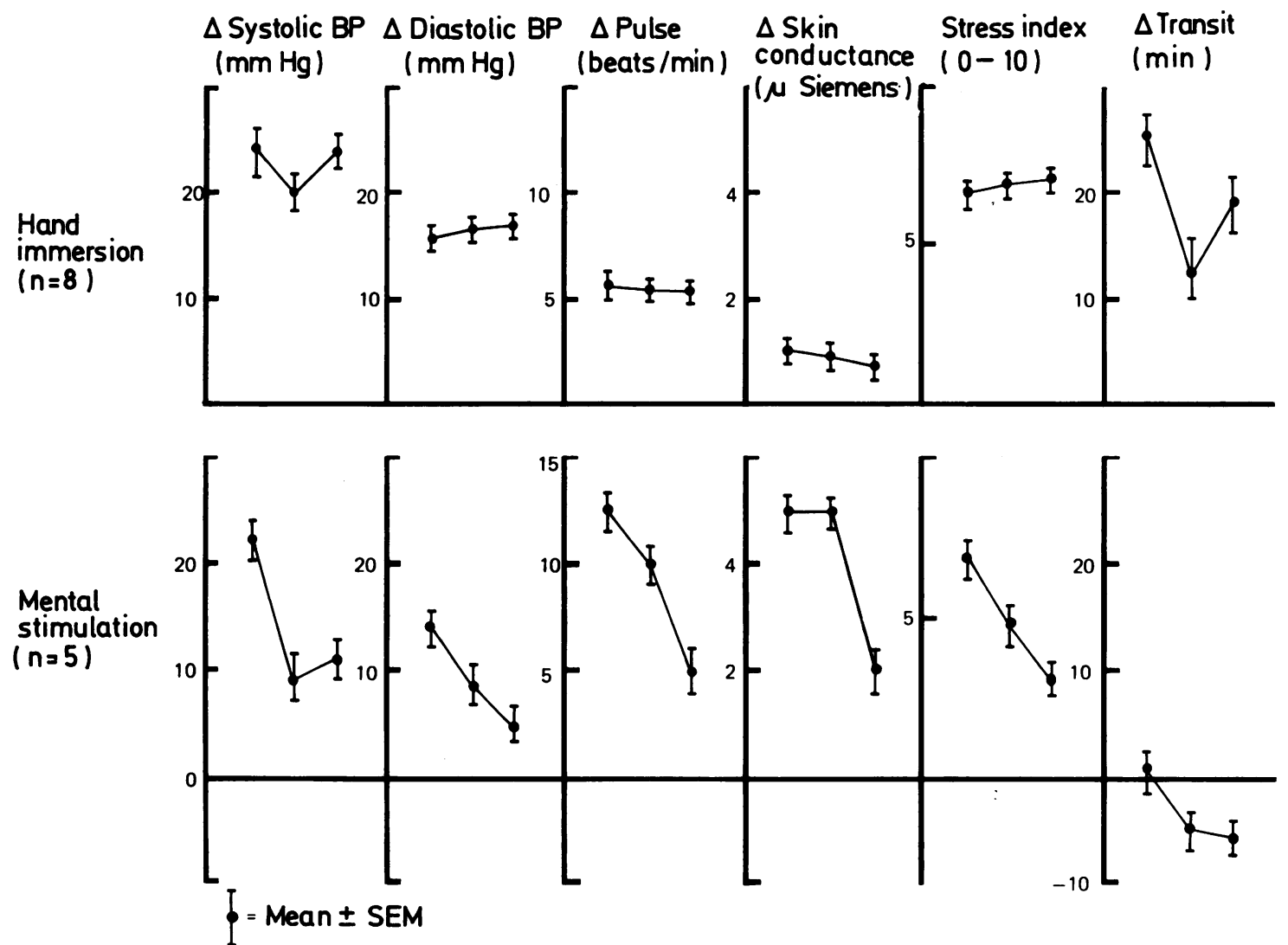

Fig. 5 Results of the repeat studies. Values shown are the mean differences between the test and control data for the paired studies. Upper graph shows consistent autonomic responses and transit delay to hand immersion in cold water, wheras mental stimulation shows a progressive reduction in automonic responses with repeated study, and no transit delay.

experiments, seems to be predominantly controlled by gastric fundal tone rather than antral peristalsis. ${ }^{33}$ Furthermore, prior administration of beta blockers to normal subjects hastens gastric emptying ${ }^{3+}$ indicating a role for beta adrenoreceptors in control of gastric tone even under non-stressful circumstances.

In contrast with the cold water induced transit delay, no consistent effects of mental stimulation were observed on transit. This lack of effect was initially a surprise in view of previously reported data indicating that a similar stimulus could accelerate transit. " There seems little doubt, however, that our stimulus was effective in disturbing autonomic function, all subjects reported stressful disturbance, and all showed measurable extra-intestinal autonomic responses which were equivalent to, or exceeded, those induced by the hand immersion in cold water. Furthermore comparison of our extraintestinal responses with those of other workers using this stimulus ${ }^{711}$ indicates that their magnitude was at least as great if not greater than those achieved by others. In seeking to explain these differences, a number of possibilities arise. First, as indicated by the extra-intestinal responses, our mental stimulus could have been more severe than in previous experiments. This might have delayed gastric emptying as in the cold immersion experiments in addition to accelerating small intestinal transit. A combination of factors which may then have resulted in no overall effect. Against this hypothesis however, we failed to show any acceleration of transit in those individuals who responded least to the stimulus. Another important factor may have been differences in test meal composition between the studies. The transit marker used in our study (lactulose) remains largely unaffected by intraluminal digestion and is poorly absorbed.$^{35}$ In contrast, transit markers such as starch, ${ }^{36}$ or stachyose ${ }^{11}$ which were used in other experiments may be variably digested and absorbed, 

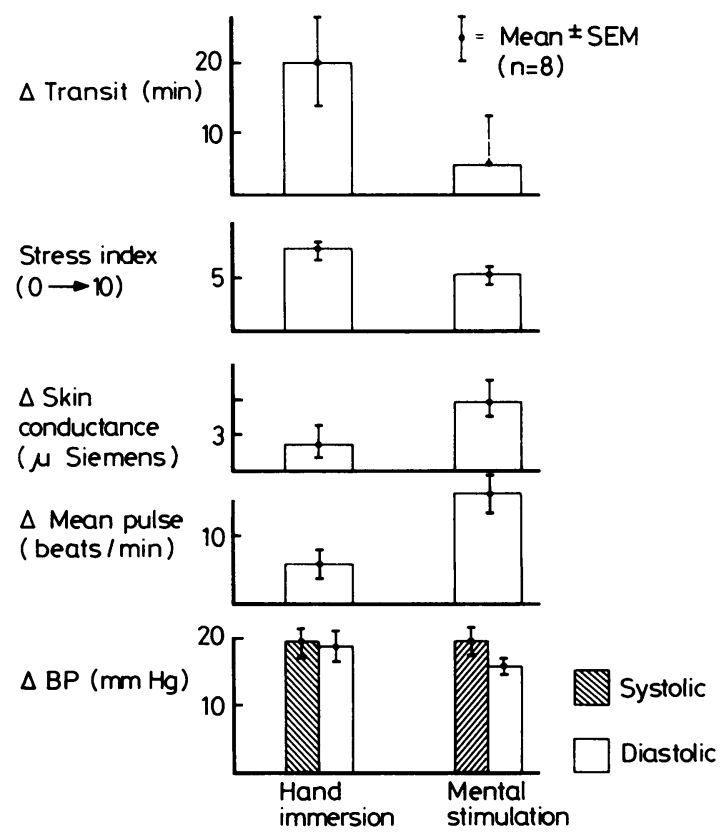

Fig. 6 Data from eight individuals who repeated both experiments. Pressor responses to the two stimuli were comparable, but mental stimulation induced significantly greater changes in pulse rate and skin conductance. Hand immersion in cold water was recorded as the more unpleasant stimulus, producing a significant delay in transit.

rendering their transit more liable to influence by stress induced changes in pancreatic secretion, ${ }^{+}$and small intestinal absorption. ${ }^{2 \times 24}$

The results of the studies which exposed individuals to both stimuli provide further support for the existence of stimulus dependent differences in autonomic response. The pattern of this difference, with similar pressor but different pulse and skin conductance responses seems to indicate that qualitative rather than quantitative differences are operating. This finding therefore indicates that differences in data interpretation are likely to arise when only single parameter is used as an index of the stress response. But the choice of parameter or combination of parameters is not certain. It is sometimes suggested that catecholamines should become the 'gold standard' for the stress response. Certainly many experimental stressors are associated with an elevation in circulating catecholamines, ${ }^{37}$ but it is important to recognise that changes in circulating levels of catecholamines correlate poorly with other autonomic variables such as blood pressure ${ }^{3 *}$ and that noradrenaline levels obtained from an antecubital vein are largely the result of spillover from noradrenergic synapses in forearm muscle. ${ }^{38}$ Furthermore, as we have shown with the mental stimulation experiments, lesser degrees of sympathetic stimulation, may induce autonomic responses without detectable changes in circulating noradrenaline.

The results of the repeat studies show additional differences between the two stimuli. Habituation to the mental stimulation task rapidly occurred, making repeated studies on the same individual difficult. In contrast, cold water stimulation did not lose its effect over three consecutive studies, making it possible to design future experiments of the pharmacological mechanisms using individuals as their own controls.

Our studies lead to a number of conclusions. It seems obvious that care should now be taken when attempting to relate the results of one autonomic stimulus on the gut with that obtained using a different technique. Similar caution should prevail in the use of the word 'stress' in the context of experimental gastroenterology, as it can no longer be taken to convey any specific meaning in terms of autonomic response either within or outside the gut. For future studies it seems necessary for the term to be qualified each time it is used by a description of the stimulus used - for example stress induced by hand immersion in cold water, stress induced by dichotic listening. In addition, the precise method of stimulus application, the prior experience of the subject, and the duration of the stimulation all need to be specified.

Marked genetic polymorphism exists in all structure and biological activities of those enzymes responsible for catecholamine synthesis, release, and metabolism, ${ }^{39}$ and the density and sensitivity of tissue adrenoreceptors shows similar variation." It is thus conceivable that some individuals may by physiologically predisposed to exhibit more florid sympathetic responses or show different degrees of organ specificity in response, which may render them more susceptible to gastrointestinal disease. The application of these experimental stimuli to studies of patients suspected to suffer from autonomically mediated disorders of the upper gut is thus an obvious next step. Recent reports already suggest that irritable bowel patients may have an exaggerated systemic autonomic response to cold water stimulation," and show a more florid gut response to mental stress. ${ }^{12}$ The development of symptoms in such patients might therefore be related to excessive disturbance of gut autonomic function.

\section{References}

1 Beaumont W. In: Experiments and observations on the gastric juice and the physiology of digestion. Plattsburg: FP Allen, 1983.

2 Wolf S, Wolff HG. Human gastric function. New York, Oxford University Press, 1943. 
3 Wolf S. The psyche and the stomach. A historical vignette. Gastroenterology 1981; 80: 605-14.

4 Thompson DG, Richelson E, Malagelada JR. Pertubation of upper gastrointestinal function by cold stress. Gut 1983; 24: 277-83.

5 Stanghellini V, Malagelada JR, Zinmeister AR, Go VLW, Kao PC. Stress-induced gastroduodenal motor disturbance in humans: Possible humoral mechanisms. Gastroenterology 1983; 85: 83-91.

6 Thompson DG, Richelson E, Malagelada JR. Pertubation of gastric emptying and duodenal motility through the central nervous system. Gastroenterology 1982; 83: 1200-6.

7 McRae S, Younger K, Thompson DG, Wingate DL. Sustained mental stress alters human jejunal motor activity. Gut 1982; 23: 404-9.

8 Valori RM, Patrick MPH, Raiman A, Parnham AP, Wingate DL. Prolonged and intermittent stress inhibits human fasting motor complexes. [Abstract] Gut 1982; 23: A914.

9 Erckenbrecht JF, Ziemer B, Lesch M, et al. Effect of long term mental stress by noise on transit of a meal through the small and large bowel. [Abstract] Gut 1984; 25: A1311.

10 Cann PA, Read NW, Cammack J, et al Psychological stress and the passage of a standard meal through stomach and small intestine in man. Gut 1983; 24: 236-40.

11 Selye H. Stress in health and disease. Boston: Butterworths, 1976.

12 Sersen EA, Clausen J, Lidsky A. Autonomic specificity and stereotypy revised. Psychophysiology 1978; 15: 60-67.

13 Bond JH, Levitt MD. Investigation of small bowel transit time in man utilizing pulmonary hydrogen $(\mathrm{H} 2)$ measurements. J Lab Clin Med 1975; 85: 546-55.

14 Thompson DG, Binfield P, De Belder A, O'Brien J, Warren $S$, Wilson $M$. Extraintestinal influences on exhaled breath hydrogen measurements during the investigation of gastrointestinal disease. Gut 1985; 26: 1349-52.

15 Exhaled hydrogen monitor, operation and maintenance handbook. Renfrewshire Scotland: GMI Ltd.

16 Hines EA, Brown GE. A standard stimulus for measuring vasomotor reactions: Its application in the study of hypertension. Mayo Clin Proc 1932; 7: 332-5.

17 Lindsay PH, Norman DA. The phenomena of attention. In: Human information processing. 2nd ed. New York: Academic Press, 1977.

18 Siegel S. Non-parametric statistics for the behavioral sciences. New York: McGraw-Hill, 1956.

19 Cannon WB. Bodily changes in pain hunger fear and rage. New York: Appleton, 1929.

20 Burnstock G. The purinergic nervous system. Pharmacol Rev 1972; 24: 509-81.

21 Burnstock G. Autonomic innervation and transmission. Br Med Bull 1979; 35: 255-62.

22 Guillemin R, Vargo T, Rossier J, et al. Beta endorphin and adrenocorticotrophin are secreted concomitantly by the pituitary gland. Science 1977; 197: 136-9.

23 Vivcos $\mathrm{OH}$, Diliberto EJ, Hazum E, Chang $\mathrm{K}$. Enkephalins as possible adrenomedullary hormones, storage secretion and regulation of synthesis. In: Casta E, Trabucchi M, eds. Neural peptides and neuronal communication. New York: Raven Press, 1980.

24 Tigranian RA, Orloff LL, Kalita NF, Davydova NA, Pavlova EA. Changes of blood levels of several hormones catecholamines prostaglandins electrolytes and c AMP in man during emotional stress. Endocrinol $\operatorname{Exp} 1980$; 14: 101-12.

25 Lacey JI, Van Lehn R. Differential emphasis in somatic response to stress. Psychosom Med 1952; 14: 71-81.

26 Wolff $\mathrm{HH}$. The mechanism and significance of the cold pressor response. $Q J$ Med 1951; 20: 261-273.

27 Lovallo W. The cold pressor test and autonomic function. A review and integration. Psychophysiology $1975 ; 12: 268-82$.

28 Barclay GR. Turnberg LA. Influence of physical and psychological stress on fluid and electrolyte absorption in the human jejunum in vivo. [Abstract] Gut 1985: 26: A554.

29 Woodmansey P, Bates TE, Read NW. Effect of psychological stress on fluid and electrolyte transport in the human jejenum. [Abstract] Gut 1983; 24: A991.

30 Lands AM, Arnold A, McAuliff JP, Ludvena FP, Brown TG. Differentiation of receptor systems activated by sympathomimetic amines. Nature 1967; 214: 597-8.

31 Gillespie JS, Khoyi MA. The site and receptors responsible for the inhibition by sympathetic nerves of intestinal smooth muscle and its parasympathetic motor nerves. J Physiol 1977; 267: 767-89.

32 Hedges A, Turner P. Beta receptors in human isolated smooth muscle. Br J Pharmacol 1969; 37: 547-8.

33 Hinder RA, Kelly KA. Canine gastric emptying of solids and liquids. Am J Physiol 1977; 233: E335-40.

34 Rees MR, Clark RA, Holdsworth CD. The effect of Beta adrenoreceptor agonists and antagonists on gastric emptying in man. Br J Pharmacol 1980; 10: 551-4.

35 Elkington SE. Lactulose. Gut 1970; 11: 1045-8.

36 Levitt MB. Malabsorption of starch. A normal phenomenon. Gastroenterology 1983; 85: 769-70.

37 Robertson D, Johnson GA, Robertson RM, Nies AS, Shand DG, Oates JA. Comparative assessment of stimuli that release neuronal and adrenomedullary catecholamines in man. Circulation 1979; 59: 637-43.

38 Silverberg AB, Shah SD, Haymond MW, Cryer PE. Norepinephrine: Hormone and neurotransmitter in man. Am J Physiol 1978; 234: E252-6.

39 Weinshilboum RM. Biochemical genetics of catecholamines in humans. Mayo Clin Proc 1983; 58: 319-330.

40 Lefkowitz RJ. Clinical physiology of adrenergic receptor regulation. Am J Physiol 1981; 243: E43-7.

41 Fielding JF, Regan R. Excessive cold pressor responses in the irritable bowel syndrome. Irish J Med Sci 1984: 153: 348-50.

42 Kumar D, Wingate DL. The irritable bowel syndrome, a paroxysmal motor disorder. Lancet 1985; ii: 973-7. 